\title{
ANÁLISE DO DESEMPENHO DAS ESCOLAS DE ENSINO MÉDIO ATRAVÉS DA METODOLOGIA DEA (DATA ENVELOPMENT ANALYSIS)
}

\section{PERFORMANCE ANALYSIS OF MIDDLE SCHOOLS TEACHING THROUGH THE METHODOLOGY DEA (Data Envelopment Analysis)}

Sebastião Geraldo Barbosa; Carlos Ropelatto Fernandes; Marilene M. Yamamoto Pires

UNESPAR- Universidade Estadual do Paraná, Paranavaí - PR, Brasil. E-mail: sebastiaogbarbosa@gmail.com

RESUMO - Este trabalho teve como objetivo analisar o desempenho das escolas de ensino médio pertencentes aos Núcleos Regionais de Educação de Paranavaí, Loanda e Cianorte, aplicando a metodologia DEA (Data Envelopment Analysis) e regressão Tobit. Através dos vários modelos DEAs procurou-se determinar os índices de eficiência e o ranking das escolas, identificando as DMUs (Decision Making Unit) pertencentes ao Benchmarking, com a finalidade de propor linhas de ações para as escolas ineficientes. Através da regressão Tobit procurou-se analisar o grau de relacionamento entre os índices de eficiências das escolas e os insumos, identificando entre os vários modelos DEAs, o mais consistente para este tipo de pesquisa. Todo o trabalho foi fundamentado nos dados obtidos através dos questionários respondidos pelos candidatos e resultados dos seis últimos vestibulares da UNESPAR - Campus de Paranavaí.

Palavras-chaves: Eficiência; Análise; Desempenho.

ABSTRACT - This study aimed to analyze the performance of high schools using as sample size the schools belonging to the Regional Education Centers Paranavaí, Loanda and Cianorte applying the methodology DEA (Data Envelopment Analysis) and Tobit regression. Through the various models DEAs were determined efficiency ratios and the ranking of schools seeking to identify the DMUs (Decision Making Unit) belonging to Benchmarking for the purpose of proposing lines of actions for educational institutions ineffective. All work was based on data obtained from questionnaires answered by the candidates and results of the last four of the vestibular UNESPAR/FAFIPA. Tobit regression was sought to analyze the degree of relationship between the indices of efficiency of schools and inputs, trying to identify between the various models DEAs, the most consistent for this type of research.

Keywords: Efficiency; Analysis; Performance.

Recebido em: 22/04/2014

Revisado em: 02/11/2014

Aprovado em: 04/11/2014_ 


\section{INTRODUÇÃO}

Os instrumentos utilizados pelo MEC para avaliação das escolas públicas (Enem, Ideb) apenas diagnostica a (in)eficiência do processo sem levar de maneira objetiva as diversas realidades vivenciadas pelas escolas. Importante, seria um processo que possa diagnosticar de maneira mais eficaz as causas dos insucessos dos alunos nas instituições públicas levando em consideração todas as diversidades das unidades educacionais.

Na busca de uma nova metodologia, alguns pesquisadores têm desenvolvido novas técnicas para analisar o desempenho das unidades de ensinos e fornecer subsídios para as tomadas de decisões. Dentre os vários modelos presente na literatura destacam-se DEA (Data Envelopment Analysis), desenvolvido por Charnes, Cooper e Rhodes (1978).

Os vários modelos DEAs permitem determinar índices de eficiências das instituições de ensino levando em consideração as variáveis significativas (insumos) ao dia a dia da escola que interferem no desempenho (produtos) dos alunos. Por exemplo, uma escola pode obter um alto índice de aprovação, porém todas as variáveis (insumos) são favoráveis enquanto, outra pode apresentar um menor índice, porém as variáveis podem ser desfavoráveis, o que pode indicar um melhor índice de eficiência mesmo não apresentando o melhor resultado final.

A análise de regressão Tobit auxilia este processo, no sentido de verificar e medir a consistência dos modelos DEAs utilizados, uma vez que os índices determinados pela metodologia DEA apresentam alguns intervalos de truncamentos ou censurados.

$\mathrm{Na}$ literatura encontram-se vários trabalhos que empregam DEA e Regressão Tobit. Por ex., Kirjavainen e Loikkanen (1995) realizaram um trabalho para avaliar 291 escolas secundárias da Finlândia, utilizando a Regressão Tobit e DEA para explicar o grau de ineficiência das escolas.

Rios (2005), realizou um trabalho, em sua dissertação de mestrado, medindo a eficiência relativa das operações dos terminais de contêineres do Mercosul, utilizando Data Envelopment Analysis e Análise de Regressão Tobit.

O que se busca através deste projeto é apresentar algumas ferramentas que servem de instrumento para analisar e medir o desempenho das escolas, de maneira mais justa levando em consideração as suas diversidades.

\subsection{DATA ENVELOPMENT ANALYSIS - DEA}

O DEA (Análise Envoltória de Dados) envolve o uso de métodos de programação linear para construir uma fronteira nãoparamétrica sobre os dados, onde as medidas 
de eficiências são calculadas em relação a sua fronteira.

O DEA é considerado, hoje, como uma poderosa ferramenta para definir estratégias para o Benchmarking, com a finalidade de indicar linhas de ações para tornar eficientes empresas ineficientes. Benchmarking pode ser considerado como um grupo de DMUs eficientes que servem de referência para que outras DMUs ineficientes consigam suas eficiências.

São várias as formulações dos modelos de DEA encontrados na literatura, porém são dois os modelos básicos mais utilizados nas aplicações. O modelo chamado CCR (CHARNES; COOPER; RHODES, 1978), também conhecido como CCR (Constante Returnes to Scale), avalia a eficiência total, identifica as DMUs eficientes e determina a que distância da fronteira de eficiência estão as unidades ineficientes. $\mathrm{O}$ modelo chamado BCC (BANKER; CHARNES; COOPER, 1984), conhecido como VRS (Variable Returns to Scale), utiliza a função dual, permitindo a projeção de cada DMU ineficiente sobre a superfície de fronteira de produção determinada pelas DMUs eficientes.

- Modelo CCR - Modelo dos multiplicadores, com retorno constante de escala.

\section{a) Orientação insumo}

Quando um único insumo é usado para gerar um único produto, a produtividade do plano de operação observado $(x, y)$ é calculado por $\mathrm{EF}=\frac{x}{y}$.

Porém, quando existem vários insumos para gerar vários produtos, a eficiência EF pode ser calculada pela razão entre as quantidades dos produtos gerados agregados em um único produto virtual e as quantidades dos insumos consumidos agregadas em único insumo virtual.

$$
\begin{aligned}
E F C\left(X_{0} Y_{0}\right)=\max & \sum_{k=1}^{M} u_{k} y_{o k} \\
\text { S.a } & \sum_{i=1}^{N} v_{i} x_{0 i}=1 \\
& \sum_{i=1}^{M} u_{k} y_{j k}-\sum_{i=1}^{N} v_{i} x_{j i} \leq 0, \\
& j=1, \ldots, S \\
& u_{k} \geq 0, \forall k, \\
& v_{i} \geq 0, \forall i
\end{aligned}
$$

Onde:

S é o número de produtores;

$N$ é o número de insumos;

$M$ é o número de produtos;

$x_{o \mathrm{i}}$ é a quantidade de insumo i da o-ésima $\mathrm{DMU}$;

$y_{o k}$ é a quantidade de produto $k$ da o-ésima $\mathrm{DMU}$;

$u_{\mathrm{k}}$ é o peso atribuído ao produto k;

$v_{\mathrm{i}}$ é o peso atribuído ao insumo i;

$y_{j k}$ é a quantidade de produto k observado da DMU j;

$x_{j i}$ é a quantidade de insumo i observado da DMU j.

\section{b) Orientação produto}

Em algumas situações, conforme Coelli, Rao e Baltese (1998), torna-se mais interessante selecionar modelos com 
orientação produção. Segundo eles algumas unidades poderiam ter uma quantidade fixada de recursos pretendendo produzir muitos produtos. Neste caso, uma orientação produto poderia ser mais conveniente onde o objetivo é maximizar os produtos obtidos sem alterar o nível atual de insumos. Serão dadas as quantidades consumidas dos insumos e a eficiência técnica será calculada a partir da máxima expansão radial dos vetores dos produtos. $\mathrm{O}$ objetivo é maximizar os produtos obtidos sem alterar o nível atual dos insumos.

$$
\begin{aligned}
E F C\left(X_{0} Y_{0}\right)= & \min \sum_{i=1}^{N} v_{i} x_{i 0} \\
\text { S. } a \quad & \sum_{k=1}^{M} u_{k} y_{k 0}=1 \\
& \sum_{i=1}^{N} v_{i} x_{j i}-\sum_{k=1}^{M} u_{k} y_{j k} \geq 0, j=1, \ldots, s \\
& u_{k} \geq 0, \forall k, \\
& v_{i} \geq 0, \forall i
\end{aligned}
$$

- Modelo BCC - Modelo dos multiplicadores, com retorno variável de escala

Outro modelo, considerado fundamental para o desenvolvimento da metodologia DEA é denominado de modelo BCC (BANKER; CHARNES; COOPER, 1984), conhecido como VRS (Variable Returns to Scale), pois pressupõe retornos variáveis de escala.

a) Orientação insumo

$$
\begin{aligned}
& E F C_{c}\left(X_{0} Y_{0}\right)=\max \sum_{k=1}^{M} u_{k} y_{o k}+u^{\prime} \\
& \text { s.a } \quad \sum_{i=1}^{N} v_{i} x_{0 i}=1 \\
& \sum_{i=1}^{M} u_{k} y_{j k}-\sum_{i=1}^{N} v_{i} x_{j i}+u^{\cdot} \leq 0, \quad j=1, \ldots, S \\
& u_{k} \geq 0, \forall k, \quad v_{i} \geq 0, \forall i, \quad u^{\prime} \text { irrestrito }
\end{aligned}
$$

b) Orientação produto:

$$
\begin{gathered}
\text { EFC }\left(x_{0} Y_{0}\right)=\min \sum_{i=1}^{N} v_{i} x_{i 0}+v^{*} \\
\text { S.a } \quad \sum_{k=1}^{M} u_{k} y_{k 0}=1 \\
\sum_{i=1}^{N} v_{i} x_{j i}-\sum_{k=1}^{M} u_{k} y_{j k}+v^{*} \geq 0, j=1, \ldots, S \\
u_{k} \geq 0, \forall k, \quad v_{i} \geq 0, \forall i, \quad v^{*} \text { irrestrito }
\end{gathered}
$$

\subsubsection{MEDIDAS DE EFICIÊNCIAS COMPLETAS}

$$
\text { Encontram-se disponíveis na }
$$

literatura várias medidas completas, mas as mais conhecidas são:

- Medida baseada em folgas - SBM (Slack

\section{Based Measure)}

Este modelo foi proposto por Tone e Tsutsui (2010) e é baseada nos excessos de consumo e nas folgas de produção relativamente às quantidades observadas. Para todo plano de produção observado, a medida é:

$$
\begin{gathered}
\tau^{*}\left(x_{o}, y_{o}\right)=\min \tau \frac{1-\frac{1}{N} \sum_{n=1}^{N} \frac{s_{n}}{x_{o n}}}{1+\frac{1}{M} \sum_{m=1}^{M} \frac{t_{m}}{y_{o m}}} \\
\text { s. } a \quad \sum_{j=1}^{S} \lambda_{j} x_{j n}+s_{n}=x_{o n}, \\
n=1, \ldots, N \\
\sum_{j=1}^{S} \lambda_{j} y_{j m}-t_{m}=y_{o m} \\
m=1, \ldots, M \\
\lambda_{j} \geq 0 \quad \forall j ; \quad s_{n} \geq 0 \forall n ; \quad t_{m} \geq 0 \quad \forall m
\end{gathered}
$$

Onde $s_{s}$ e $t_{m}$ são vetores de quantidades de excessos de consumo e folgas na produção, respectivamente, e $\mathrm{N}$ e $\mathrm{M}$ o números de insumos e de produtos respectivamente. 
- Medida ajustada por amplitude - RAM (Range Adjusted Measure)

A medida ajustada por amplitude é calculada com base na amplitude das quantidades de insumos e produtos. Este modelo foi proposto por Cooper, Park e Pastor (1999).

$$
\begin{aligned}
\Gamma\left(x_{o}, y_{o}\right)= & \min \Gamma=1-\frac{1}{N+M}\left(\sum_{n=1}^{N} \frac{s_{n}}{R_{n}}+\sum_{m=1}^{M} \frac{t_{m}}{R_{m}}\right) \\
\text { s.a. } & \sum_{j=1}^{J} \lambda_{j} x_{j n}+s_{n}=x_{o n}, \quad n=1, \ldots, N \\
& \sum_{j=1}^{J} \lambda_{j} y_{j m}-t_{n}=y_{o m}, \quad m=1, \ldots, M \\
& \sum_{i=1}^{n} \lambda_{j}=1 \\
& \lambda_{j} \geq 0 \forall y ; \quad s_{n} \geq 0 \forall n ; \quad t_{m} \geq 0 \forall m
\end{aligned}
$$

onde

$$
\begin{aligned}
& R_{n}=\underline{x}_{n}-\bar{x}_{n} \quad \text { e } \quad R_{m}=\underline{y}_{m}-/ \bar{y}_{m} \\
& \left.\underline{x}_{n}=\min \left\{x_{j n}\right\}, \quad \bar{x}_{n}=\operatorname{maxx} \quad j n\right\}, \\
& \underline{y}_{m}=\min \left\{y_{j m}\right\}, \quad \bar{y}_{m}=\max \{y \quad j m\}, \quad \forall j
\end{aligned}
$$

\subsubsection{AVALIAÇÃO CRUZADA - (CROSS EVALUATION)}

A avaliação cruzada utiliza os escores de eficiências determinadas através do modelo tradicional DEA e seus pesos ótimos para atingirem a eficiência

No trabalho de Doyle e Green (1994) foi sugerida uma função objetivo definida que minimiza a soma ponderada dos produtos da DMU composta dividida pela soma ponderada dos inputs da DMU composta.

$$
\begin{gathered}
\min \mathrm{C}_{\mathrm{k}}=\min \sum_{i=1}^{M}\left(u_{s r} \sum_{j=1}^{S} y_{j r}\right)(j \neq S) \\
\text { s.a } \quad \sum_{i=1}^{N}\left(v_{s i} \sum_{j=1}^{S} x_{j i}\right)=1
\end{gathered}
$$

Onde $E_{k k}$ é a eficiência calculada pelo modelo $D E A$ padrão e $E_{k s}$ é a eficiência da DMUs calculada utilizando os pesos obtidos para a DMU k, $e_{k}$ é a eficiência cruzada média da DMU k. O valor de $e_{s}$ pode ser calculado através de:

$e_{s}=\frac{\sum_{k=1}^{j} E_{k s}}{s} \operatorname{com~} k \neq s$

Ou sem a diagonal principal

$\mathrm{e}_{\mathrm{s}}=\frac{\sum_{\mathrm{k}=1}^{\mathrm{j}} \mathrm{E}_{\mathrm{ks}}}{\mathrm{s}-1} \operatorname{com} \mathrm{k} \neq \mathrm{s}$

Para determinar as DMUs com a maior diferença relativa entre a eficiência padrão e a eficiência cruzada média, utilizase a fórmula:

$M_{k}=\frac{\left(E_{k k}-e_{s}\right)}{e_{s}}$

As DMUs com maiores $M_{k}$ são denominadas "Mavericks" (DOYLE; GREEN, 1994). Quando uma DMU é eficiente no modelo padrão e tem um $M_{k}$ alto é chamada de "falso positivo", mostrando aquela DMU que atinge $100 \%$ de eficiência, porém, utilizando somente pesos irreais. 


\subsection{REGRESSÃO TOBIT}

Uma amostra na qual a informação sobre a variável resposta esteja disponível somente para algumas observações é conhecida como amostra censurada. Por isto o modelo de Tobit é também chamado de regressão censurada.

Vários autores têm utilizado os modelos de regressão com o método DEA para analisar a eficiência das organizações. Thanassoulis (1993) foi um dos primeiros autores a mostrar as vantagens na aplicação de regressão no modelo DEA como métodos alternativos que podem ser utilizados para comparar a eficiência de unidades.

Em DEA, os escores de eficiência situam-se entre 0 e 1 , tornando-se problemática a aplicação dos métodos de mínimos quadrados ordinários (MQO), devendo, portanto, ser utilizado o modelo Tobit.

Matematicamente pode-se expressar o modelo Tobit pela equação:

$$
y^{*}=\beta_{o}+\beta_{1} \cdot x_{1}+\ldots+\beta_{n} x_{n}+\varepsilon
$$

A equação de Tobit pode ser considerada como um modelo de regressão linear, no qual $y^{*}$ (variável latente) substitui o y. Esta substituição é necessária devido ao modelo Tobit possuir dados censurados ou truncados. Este $y^{*}$ pode assumir valores a direita, a esquerda ou em um determinado intervalo.

$$
Y= \begin{cases}y^{*}, & \text { se } y^{*}>0 \\ 0, & \text { se } y^{*} \leq 0\end{cases}
$$

O modelo censurado, padrão Tobit, é mais facilmente definido como um modelo de variável latente, conforme:

$\left.y^{*}=x \beta+\varepsilon, \quad u / x \sim N 0, \sigma^{2}\right)$

onde:

$\beta=\left[\begin{array}{l}\beta_{0} \\ . . \\ \beta_{n}\end{array}\right]$ e $y=\max \left(0, y^{*}\right)$

Na metodologia a DEA os escores de eficiências para a orientação insumo estão situados entre 0 e 1 , portanto, existe uma distribuição não normal, mas censurada para valores menores que 0 e maior que 1 , da mesma forma, na orientação produto existe uma distribuição normal, mas censurada menores que 1 , portanto, a regressão Tobit torna-se muito útil na metodologia DEA.

Quando se pretende determinar o grau de relacionamento entre cada produto com os índices de eficiências das DMUs utilizadas em um trabalho de pesquisas, com a finalidade de verificar a consistência entre os vários modelos DEAs é conveniente a utilização de Analise de Regressão Tobit, através dos seguintes modelos:

$Y= \begin{cases}y^{*}, & \text { se } 0<y^{*} \leq 1 \\ 0, & \text { em caso contrário }\end{cases}$

para a orientação insumo 
$Y= \begin{cases}y^{*}, & \text { se } y^{*} \geq 1 \\ 0, & \text { se } y^{*}<1\end{cases}$

para orientação produto

\section{MATERIAL E MÉTODO}

As escolas de Ensino Médio, objeto de estudo deste trabalho, pertencem aos Núcleos ${ }^{1}$ de Ensino de Paranavaí, Loanda e Cianorte envolvendo 21 municípios do NRE de Paranavaí, 11 municípios do NRE de Loanda e 11 do NRE de Cianorte totalizando 68 escolas públicas com aproximadamente 20000 alunos e 06 escolas privadas com aproximadamente 2000 alunos.

Para desenvolver 0 presente estudo, foram selecionadas variáveis com maior representatividade focada na situação sócio econômica e que pudesse ter alguma influência no desempenho dos alunos nos processos seletivos das universidades. Estas variáveis foram organizadas em dois grupos, insumo e produtos conforme suas características.

\section{Insumos}

$\mathrm{X}_{1}$. Escolaridade da família;

$X_{2}$. Situação econômica da família (salarial);

$X_{3}$. Índices porcentuais dos alunos que não possui a cor da pele "branca";

\footnotetext{
${ }^{1}$ No Paraná existem 32 NRE, subordinados à SEED, coordenando e oferecendo suporte educacional às escolas de ensino básico em 395 municípios do Estado. Eles acompanham a implantação e execução das políticas educacionais do Estado do Paraná nas escolas de sua jurisdição.
}

$X_{4}$. Índices porcentuais de alunos que não trabalham;

$X_{5}$. Índices que medem o tempo que $o$ candidato fez ensino fundamental em escola privada;

$\mathrm{X}_{6}$. Índices que medem o tempo que o candidato fez Ensino Médio em escola privada

\section{Produtos}

$Y_{1}$. Índice porcentual dos alunos inscritos;

$\mathrm{Y}_{2}$. Índice porcentual dos alunos classificados no limite de vagas;

$\mathrm{Y}_{3}$. Índice porcentual dos alunos aprovados ${ }^{2}$.

Os levantamentos dos dados foram obtidos através de questionários respondidos pelos candidatos no momento da inscrição e relatórios obtidos pelo sistema do vestibular da UNESPAR/FAFIPA. Foram utilizados como amostra, os resultados dos quatro últimos concursos vestibulares da FAFIPA, envolvendo aproximadamente 7200 candidatos.

Para a tabulação dos dados foi utilizado o software Sphiny Léxica e lançados para o Microsoft Excel para a construção das tabelas. Para determinar os índices das variáveis $X_{1}$, e $X_{2}$ foram utilizados pesos nas alternativas dos questionários de forma a maximizar os valores. Por exemplo, maior índice corresponde mais escolarização e

\footnotetext{
2 Consideram-se candidatos aprovados todos que conseguiram nota da redação maior ou igual a 3.0 e média final maior ou igual a 2.0.
} 
melhor situação econômica. Houve, também, uma preocupação no sentido de direcionar as variáveis insumos com os produtos trabalhados (ex: partiu-se do princípio que a escola com clientela de maior escore salarial tem melhor desempenho) e também manter a mesma escala para todas as variáveis.

Das 72 escolas pesquisadas foram descartadas 22 em função do pequeno número de candidatos inscritos, com a finalidade de conseguir melhor consistência dos dados.

A tabela 1 foi obtida a partir da depuração, conversão e direcionamento das variáveis insumos/produtos.

Tabela 1. Insumos e produtos coletados no vestibular de 2010/2012 - FAFIPA

\begin{tabular}{|c|c|c|c|c|c|c|c|c|c|}
\hline Esc. & $X_{1}$ & $x_{2}$ & $X_{3}$ & $X_{4}$ & $X_{5}$ & $X_{6}$ & $Y_{1}$ & $Y_{2}$ & $Y_{3}$ \\
\hline 1. & 12.8546 & 4.47330 & 67.8600 & 51.7857 & 12.5000 & 21.4286 & 1.4733 & 14.2857 & 55.3571 \\
\hline 2. & | 10.6981 & 5.21212 & 80.0000 & 32.0000 & 4.0000 & 8.0000 & 0.6577 & 8.0000 & 64.0000 \\
\hline 3. & 15.6995 & 5.49563 & 76.2700 & 40.3226 & 6.7797 & 1.6949 & 1.5522 & 18.6441 & 66.1017 \\
\hline 4. & 13.9911 & 6.09169 & 73.8500 & 52.3077 & 0.0001 & 0.0001 & 1.7101 & 16.9231 & 67.6923 \\
\hline 5. & 11.1012 & 5.05051 & 58.3300 & 41.6667 & 8.3333 & 8.3333 & 0.3157 & 8.3333 & 83.3333 \\
\hline 6. & 13.5595 & 4.79798 & 73.0800 & 48.0769 & 3.8462 & 3.8462 & 1.3681 & 17.3077 & 76.9231 \\
\hline 7. & 14.4008 & 5.16595 & 48.6800 & 54.2056 & 18.4211 & 8.4112 & 2.8150 & 21.4953 & 73.8318 \\
\hline 8. & 14.6441 & 6.29504 & 69.2500 & 37.7143 & 13.1805 & 6.9164 & 9.1818 & 20.0573 & 71.6332 \\
\hline 9. & 11.5746 & 5.00000 & 70.0000 & 60.0000 & 0.0001 & 0.0001 & 0.5262 & 10.0000 & 55.0000 \\
\hline 10. & 10.7017 & 4.16935 & 75.4400 & 31.5789 & 1.7544 & 1.7544 & 1.4996 & 12.2807 & 61.4035 \\
\hline 11. & 4.3507 & 4.30473 & 71.9600 & 48.5981 & 8.4112 & 9.3458 & 2.8150 & 11.2150 & 67.2897 \\
\hline 12. & 3.4439 & 6.04507 & 78.4600 & 35.3846 & 1.5385 & 0.0001 & 1.7101 & 18.4615 & 83.0769 \\
\hline 13. & 1.9318 & 7.16253 & 90.9100 & 45.4545 & 9.0909 & 9.0909 & 0.2894 & 27.2727 & 63.6364 \\
\hline 14. & 10.0000 & 4.44444 & 40.0000 & 40.0000 & 20.000 & 40.0000 & 0.1 & 0.0001 & 100.000 \\
\hline 15. & 11.3472 & 5.51461 & 63.5100 & 27.0270 & 10.8108 & 14.8649 & 1.9469 & 20.2703 & 59.4595 \\
\hline 16. & 9.7470 & 2.27273 & 62.5 & $25 . C$ & 12.5000 & 12.5000 & 0.2105 & 25.0000 & 75.0000 \\
\hline 17. & 18.6706 & 10.56015 & 40.0 & 80.0 & 80.0 & 100.000 & 0.2631 & 70.0000 & 90.0000 \\
\hline 18. & 5.6076 & 4.61593 & 58.1400 & 52.3 & 22.0930 & 4.6512 & 2.2626 & 20.9302 & 68.6047 \\
\hline 19. & 13.3178 & 6.96970 & 84.0000 & 22.0000 & 0.0001 & 0.0001 & 1.3 & 20.0000 & 70.0000 \\
\hline 20. & 8.9286 & 9.49495 & 80.0000 & 60.0000 & 40.0000 & 80.0000 & 0.1315 & 20.0000 & 60.0000 \\
\hline 21. & 2.7940 & 4.66064 & 86.2100 & 29.3103 & 10.3448 & 6.8966 & 1.5259 & 29.3103 & 65.5172 \\
\hline 22. & 2.6987 & 3.70370 & 62. & 72. & 1.9608 & 1.9608 & 1.3418 & 9.8039 & 62.7451 \\
\hline 23. & 3.3303 & 5.83 & 72.3 & 32. & 7.4468 & 9.5745 & 2.4730 & 14.8936 & 69.1489 \\
\hline 24. & 13.5134 & 5.49520 & 37.1100 & 37.4214 & 38.3648 & 8.4906 & 8.4 & 21.6718 & 84.5201 \\
\hline 25. & 11.1268 & 3.08580 & 51.6500 & 70.3297 & 8.7912 & 12.0879 & 2.3941 & 13.1868 & 58.2418 \\
\hline 26. & 14.1150 & 5.33551 & 60.0000 & 43.5685 & 19.0871 & 23.2365 & 6.3404 & 29.0456 & 75.1037 \\
\hline 27. & 14.5990 & 6.13543 & 88.8900 & 22.2222 & 11.1111 & 14.8148 & 1.1839 & 11.1111 & 80.0000 \\
\hline 28. & 17.7296 & 7.0 & 100.0 & 11.1111 & 20.0000 & 22.2222 & 0.2368 & 22.2222 & 77.7778 \\
\hline 29. & 11.8182 & 7.80533 & 100.0000 & 27.2727 & 0.0001 & 7.6923 & 0.2894 & 27.2727 & 72.7273 \\
\hline 30. & 11.7695 & 6.97888 & 91.0400 & 54.5455 & 7.4627 & 9.0909 & 0.2894 & 27.2727 & 63.6364 \\
\hline 31. & 13.7345 & 5.07560 & 1.7300 & 38.5093 & 6.7308 & 6.2112 & 4.2357 & 17.3913 & 67.0807 \\
\hline 32. & $12.9 / 51$ & 4.97005 & 66.3700 & 37.1681 & 6.1947 & 5.3097 & 2.9729 & 11.5044 & 61.9469 \\
\hline 33. & 13.3641 & 6.22253 & 65.6300 & 44.5313 & 11.7188 & 12.2137 & 3.3675 & 21.0938 & 75.0000 \\
\hline 34. & 18.2632 & 10.70560 & 66.4200 & 78.8321 & 60.5839 & 76.6423 & 3.7359 & 41.5493 & 81.6901 \\
\hline 35. & 20.6627 & 11.74242 & 62.5000 & 77.5000 & 60.0000 & 100.000 & 1.0524 & 35.0000 & 72.5000 \\
\hline 36. & 19.6429 & 7.74411 & 33.3300 & 83.3333 & | 100.000 & 66.6667 & 0.1579 & 16.6667 & 50.0000 \\
\hline
\end{tabular}




\begin{tabular}{l|l|l|l|r|r|r|r|r|r} 
37. & 19.1206 & 9.07421 & 62.8100 & 77.6860 & 56.1983 & 99.1667 & 3.1834 & 35.5372 & 75.2066 \\
38. & 12.9949 & 5.71953 & 66.2300 & 50.6494 & 0.0001 & 3.8961 & 2.0258 & 16.8831 & 58.4416 \\
39. & 11.5132 & 6.09250 & 68.4200 & 30.5263 & 22.1053 & 5.2632 & 2.4993 & 11.5789 & 62.1053 \\
40. & 13.2738 & 4.12458 & 75.0000 & 41.6667 & 0.0001 & 0.0001 & 0.3157 & 8.3333 & 33.3333 \\
41. & 12.5744 & 5.72391 & 64.7100 & 31.5789 & 2.9412 & 5.5556 & 0.9471 & 25.0000 & 69.4444 \\
42. & 13.4481 & 5.44132 & 74.4000 & 45.2381 & 3.5714 & 4.1667 & 4.4199 & 13.0952 & 69.0476 \\
43. & 14.6867 & 3.97727 & 68.7500 & 56.2500 & 3.1250 & 6.2500 & 0.9734 & 10.8108 & 64.8649 \\
44. & 8.5069 & 5.27497 & 77.7800 & 55.5556 & 0.0001 & 0.0001 & 0.2368 & 0.0001 & 66.6667 \\
45. & 10.1686 & 5.21886 & 75.0000 & 8.3333 & 4.1667 & 0.0001 & 0.6314 & 0.0001 & 50.0000 \\
46. & 17.4178 & 4.64646 & 65.0000 & 55.0000 & 0.0001 & 0.0001 & 0.5262 & 10.0000 & 80.0000 \\
47. & 12.5255 & 3.93939 & 70.0000 & 10.0000 & 10.0000 & 0.0001 & 0.2631 & 20.0000 & 50.0000 \\
48. & 14.4345 & 7.11662 & 61.3600 & 18.1818 & 9.0909 & 2.2727 & 1.1576 & 18.1818 & 75.0000 \\
49. & 13.1030 & 6.12558 & 77.1900 & 23.3918 & 5.8480 & 6.4327 & 4.4988 & 22.8070 & 60.2339 \\
50. & 0.9990 & 4.63697 & 67.7900 & 36.9128 & 5.3691 & 14.9701 & 3.9200 & 14.7651 & 70.4698 \\
\hline
\end{tabular}

Fonte: Dados coletados através dos questionários sócio-econômicos e relatórios dos vestibulares

A metodologia aplicada para obtenção dos resultados pode ser destacada em duas etapas:

- Na primeira etapa foram aplicados os vários modelos DEAs envolvendo todas as escolas para determinar o seu desempenho, sendo utilizados os softwares DEA-SAED e Siadv2;

- Na segunda etapa foi utilizado a regressão Tobit para analisar a consistência dos modelos DEA e o grau de relacionamento entre os insumos e os índices de eficiências das escolas de cada modelo DEA, através do software

Evews.5.

\section{RESULTADOS E DISCUSSÃO}

Para análise dos dados construiu-se a tabela abaixo, com os índices de desempenho das 50 escolas utilizando os 6 insumos e os 3 produtos, obtidos a partir da aplicação dos vários modelos DEAs, utilizando o software DEA-SAED e Siadv2.

Tabela 2. Índice de eficiências das escolas de Ensino Médio dos NREs de Paranavaí, Loanda e Cianorte.

\begin{tabular}{c|c|c|c|c|c|c|c|c|c|c}
\hline $\begin{array}{c}\text { Esco- } \\
\text { las }\end{array}$ & $\begin{array}{c}\text { CCR } \\
\text { Insumo }\end{array}$ & $\begin{array}{c}\text { BCC } \\
\text { Insumo }\end{array}$ & $\begin{array}{c}\text { CCR } \\
\text { Produto }\end{array}$ & $\begin{array}{c}\text { BCC } \\
\text { Produto }\end{array}$ & SBM & RAM & $\begin{array}{c}\text { CRUZADA } \\
\text { CCR Ins. }\end{array}$ & Mk & \multicolumn{2}{|c|}{$\begin{array}{c}\text { Ranking } \\
\text { escolas }\end{array}$} \\
\hline 1. & 0.6360 & 0.8300 & 1.5710 & 1.4610 & 0.3330 & 0.8700 & 0.4630 & 0.3740 & $1^{\circ}$ & 12 \\
2. & 0.8930 & 0.9740 & 1.1200 & 1.0770 & 0.3460 & 0.9440 & 0.5570 & 0.6030 & $2^{\circ}$ & 41 \\
3. & 0.8800 & 0.8850 & 1.1370 & 1.1210 & 0.6560 & 0.9470 & 0.6620 & 0.3290 & $3^{\circ}$ & 16 \\
4. & 1.0000 & 1.0000 & 1.0000 & 1.0000 & 1.0000 & 1.0000 & 0.8180 & 0.2220 & $4^{\circ}$ & 19 \\
5. & 1.0000 & 1.0000 & 1.0000 & 1.0000 & 1.0000 & 1.0000 & 0.6160 & 0.6230 & $5^{\circ}$ & 46 \\
6. & 0.9650 & 0.9660 & 1.0360 & 1.0360 & 0.8130 & 0.9710 & 0.7990 & 0.2080 & $6^{\circ}$ & 31 \\
7. & 0.9660 & 0.9680 & 1.0360 & 1.0230 & 0.7110 & 0.9410 & 0.6330 & 0.5250 & $7^{\circ}$ & 4 \\
8. & 1.0000 & 1.0000 & 1.0000 & 1.0000 & 1.0000 & 1.0000 & 0.7770 & 0.2870 & $8^{\circ}$ & 10 \\
9. & 0.8990 & 1.0000 & 1.1120 & 1.0000 & 0.5150 & 1.0000 & 0.6440 & 0.3960 & $9^{\circ}$ & 6 \\
10. & 1.0000 & 1.0000 & 1.0000 & 1.0000 & 1.0000 & 1.0000 & 0.8110 & 0.2330 & $10^{\circ}$ & 38 \\
11. & 0.8850 & 0.9020 & 1.1300 & 1.1170 & 0.6210 & 0.9180 & 0.6070 & 0.4580 & $11^{\circ}$ & 50 \\
12. & 1.0000 & 1.0000 & 1.0000 & 1.0000 & 1.0000 & 1.0000 & 0.9090 & 0.1000 & $12^{\circ}$ & 49 \\
13. & 0.9650 & 0.9670 & 1.0360 & 1.0340 & 0.3410 & 0.8990 & 0.5910 & 0.6320 & $13^{\circ}$ & 29
\end{tabular}




\begin{tabular}{|c|c|c|c|c|c|c|c|c|c|c|}
\hline 14. & 1.0000 & 1.0000 & 1.0000 & 1.0000 & 1.0000 & 1.0000 & 0.3760 & 6590 & $14^{\circ}$ & \\
\hline 15. & 0.8540 & 0.9860 & 1.1710 & 1.1600 & 0.6020 & 0.9490 & 0.6170 & 0.3830 & $15^{\circ}$ & 4 \\
\hline 5. & 0000 & 1.0000 & 1.0000 & 1.0000 & 1.0000 & 1.0000 & 0.8700 & 0.1500 & $16^{\circ}$ & \\
\hline 17. & 1.0000 & 1.0000 & 1.0000 & 1.0000 & 1.0000 & 1.0000 & 4350 & 2980 & $17^{\circ}$ & \\
\hline 3. & 0.9790 & 0.9810 & 1.0210 & 1.0140 & 0.6950 & 0.9330 & 0.5620 & 0.7410 & $18^{\circ}$ & \\
\hline & 1.0000 & 1.0000 & 1.0000 & 1.0000 & 1.0000 & 1.0000 & 0.8640 & 0.1570 & $19^{\circ}$ & \\
\hline D. & 0.4770 & 0.6070 & 2.0970 & 1.4620 & 0.0230 & 0.6800 & .2440 & 0.9540 & $20^{\circ}$ & \\
\hline & 1.0000 & 1.0000 & 1.0000 & 1.0000 & 1.0000 & 1.0000 & 0.7620 & 0.3120 & $21^{\circ}$ & \\
\hline & 1.0000 & 1.0000 & 1.0000 & 1.0000 & 1.0000 & 1.0000 & 0.7270 & 0.3750 & $22^{\circ}$ & \\
\hline & 0.8570 & 0.8940 & 1.1670 & 1.1610 & 0.5950 & 0.9380 & 0.6390 & 0.3410 & $23^{\circ}$ & \\
\hline 4. & 1.0000 & 1.0000 & 1.0000 & 1.0000 & 1.0000 & 1.0000 & 0.6740 & 0.4830 & $24^{\circ}$ & \\
\hline & 0.9750 & 1.0000 & 1.0260 & 1.0000 & 0.7090 & 1.0000 & 0.6370 & 0.5300 & $25^{\circ}$ & \\
\hline 6. & 1.0000 & 1.0000 & 1.0000 & 1.0000 & 1.0000 & 1.0000 & 0.7220 & .3840 & $26^{\circ}$ & \\
\hline 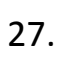 & 0.9890 & 1.0000 & 1.0110 & 1.0000 & 0.6120 & 1.0000 & 0.5350 & 0.8480 & $27^{\circ}$ & \\
\hline & 1.0000 & 1.0000 & 1.0000 & 1.0000 & 1.0000 & 1.0000 & 0.4820 & 1.0730 & $28^{\circ}$ & \\
\hline 29. & 1.0000 & 1.0000 & 1.0000 & 1.0000 & 1.0000 & 1.0000 & 0.7780 & 0.2860 & $29^{\circ}$ & \\
\hline & 0.9790 & 0.9790 & 1.0220 & 1.0200 & 0.4270 & 0.9070 & 0.6100 & 0.6050 & $30^{\circ}$ & \\
\hline 1. & 1.0000 & 1.0000 & 1.0000 & 1.0000 & 1.0000 & 1.0000 & 0.8290 & 0.2060 & $31^{\circ}$ & \\
\hline & 0.8480 & 0.9210 & 1.1800 & 1.1710 & 0.6570 & 0.9470 & 0.6 & 0.3100 & $32^{\circ}$ & \\
\hline & 0.9160 & 0.9240 & 1.0920 & 1.0620 & 0.7190 & 0.9490 & 0.6700 & 0.3660 & $33^{\circ}$ & \\
\hline 4. & 0.8080 & 0.8170 & 1.2370 & 1.0770 & 0.5330 & 0.8410 & 0.3 & 1.1400 & $34^{\circ}$ & \\
\hline 35. & 0.6260 & 0.6890 & 1.5980 & 1.2310 & 0.1560 & 0.7440 & 0.2890 & 1.1620 & $35^{\circ}$ & \\
\hline 5. & 0.6520 & 1.0000 & 1.5330 & 1.0000 & 0.0310 & 1.0000 & 0.1 & 3.1250 & $36^{\circ}$ & \\
\hline . & 0.6870 & 0.7230 & 1.4560 & 1.1800 & 0.3990 & 0.8130 & 0.3 & 1.0160 & $37^{\circ}$ & \\
\hline 8. & 1.0000 & 1.0000 & 1.0000 & 1.0000 & 1.0000 & 1.0000 & 0.7970 & 0.2540 & $38^{\circ}$ & \\
\hline 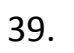 & 0.8290 & 0.9720 & 1.2060 & 1.1410 & 0.5170 & 0.9280 & 0.4480 & 0.8510 & $39^{\circ}$ & \\
\hline . & 0.7270 & 1.0000 & 1.3750 & 1.0000 & 1.0000 & 1.0000 & 0.4900 & 1.0410 & $40^{\circ}$ & \\
\hline 1 & 1.0000 & 1.0000 & 1.0000 & 1.0000 & 1.0000 & 1.0000 & 0.8770 & 0.1400 & $41^{\circ}$ & \\
\hline 42. & 1.0000 & 1.0000 & 1.0000 & 1.0000 & 1.0000 & 1.0000 & 0.7730 & 0.2930 & $42^{\circ}$ & \\
\hline 43. & 0.8920 & 0.9540 & 1.1210 & 1.1210 & 0.6030 & 0.9620 & 0.6570 & 0.3580 & $43^{\circ}$ & \\
\hline 44. & 1.0000 & 1.0000 & 1.0000 & 1.0000 & 1.0000 & 1.0000 & 0.5270 & 0.8990 & $44^{\circ}$ & \\
\hline 45. & 1.0000 & 1.0000 & 1.0000 & 1.0000 & 1.0000 & 1.0000 & 0.2310 & 3.3300 & $45^{\circ}$ & \\
\hline 46. & 1.0000 & 1.0000 & 1.0000 & 1.0000 & 1.0000 & 1.0000 & 0.8350 & 0.1980 & $46^{\circ}$ & \\
\hline 47. & 1.0000 & 1.0000 & 1.0000 & 1.0000 & 1.0000 & 1.0000 & 0.6580 & 0.5190 & $47^{\circ}$ & \\
\hline 48. & 1.0000 & 1.0000 & 1.0000 & 1.0000 & 1.0000 & 1.0000 & 0.6730 & 0.4850 & $48^{\circ}$ & \\
\hline 49. & 1.0000 & 1.0000 & 1.0000 & 1.0000 & 1.0000 & 1.0000 & 0.7920 & 0.2630 & $49^{\circ}$ & \\
\hline 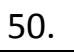 & 1.0000 & 1.0000 & 1.0000 & 1.0000 & 1.0000 & 1.0000 & 0.7940 & 0.2600 & $50^{\circ}$ & \\
\hline
\end{tabular}

Fonte: Dados obtidos através do software DEA-SAED e Siadv2.

Observa-se, pela tabela 2, que a escola 12 foi a melhor ranqueada, avaliação cruzada, isto indica que mesmo com as condições desfavoráveis (alunos de escolas públicas), apresentou um razoável desempenho no concurso vestibular. No entanto, a escola 17, por exemplo, formados por alunos de ensino privado, com $70 \%$ de aprovação no limite de vaga, ficou ranqueada em 43ำ lugar, pois as condições foram todas favoráveis (bons insumos). Isto mostra a grande vantagem do DEA: não bastam tão somente os resultados, são analisadas, também, as condições dos insumos.

Através do modelo DEA BCC insumo, pode-se observar que houve 31 
escolas com eficiência máxima, sendo que a escola 20 foi a menos eficiente com um índice de produtividade igual 0.6070 . Este modelo, mesmo considerado o mais consistente (tabela 4), apresentou várias DMUs com pesos irreais. Isto pode ser comprovado determinando o $\quad M_{k}$, "Maverick". Por ex., a DMU 12 tem $M_{k}=$ 0,1000 considerada mais eficiente pois possui menor $M_{k}$, isto significa, que sua eficiência é consistente ou seja, com pesos reais, enquanto ao DMU 36 considerada eficiente no modelo CCR produto, porém seus pesos são irreais, denominado falso positivo, pois possui $\mathrm{M}_{\mathrm{k}}=3.1250$. Isto acontece, pois os modelos DEAs tradicionais, várias DMUs, para conseguirem sua eficiência, assume pesos nulos, daí a importância do modelo de Avaliação Cruzada.

Um fato que merece atenção, conforme tabela 2 , é que as escolas 22 e 25 consideradas eficientes em todos os modelos, foram ranqueadas, através da avaliação cruzada, na 17a e 28a posição, pois seus $M_{k}$ são relativamente altos o que indica eficiência com pesos irreais.

Para cálculo dos índices de eficiência da avaliação cruzada foram utilizados os índices de eficiência do modelo BCC insumo pelo fato de ter apresentado um melhor relacionamento com os insumos (tabela 5).
Tabela 3. Benchmarking construído através do modelo BCC insumo

\begin{tabular}{c|c}
\hline DMU & Benchmarking \\
\hline 1 & $14,16,24,25,31$ e 44 \\
2 & 10,16 e 44 \\
3 & $12,16,21,22,31,41$ e 47 \\
6 & $10,12,16,22,41,46$ e 50 \\
7 & $16,17,24,31$ e 41 \\
11 & $8,10,16,22,25$ e 50 \\
13 & 16,21 e 29 \\
15 & $16,24,31,47$ e 49 \\
18 & $12,16,24,46$ e 47 \\
23 & $16,19,31,47,49$ e 50 \\
30 & $16,17,21$ e 29 \\
32 & $8,10,16,24,31,49$ e 50 \\
33 & $12,14,16,24,26,31$ e 50 \\
34 & $16,17,24$ e 26 \\
35 & $14,16,17,24$ e 31 \\
37 & $14,16,17,24$ e 31 \\
39 & $10,16,24$ e 47 \\
43 & $10,16,22,31,40$ e 46 \\
\hline
\end{tabular}

Fonte: Os índices foram obtidos pelo software Evews.5.

Observa-se pela tabela 3 que a escola 16, 3a colocada no ranking, serve de referência (Benchmarking) para as outras escolas. Esta escola não apresentou um melhor desempenho principalmente no produto $Y_{2}$ (índice de aprovação no limite de vagas), porém as condições (insumos) não foram tão favoráveis, motivo, pelo qual tornou-se uma grande referência para as demais.

As DMUs que constituem o Benchmarking servem como referência para 
aquelas que não são eficientes, conseguirem sua eficiência. Por ex., a escola 2, não eficiente, pode conseguir sua eficiência, espelhando-se nos projetos desenvolvidos pelas escolas 10, 16 e 44. Observa-se que a escola 16 é uma das melhores ranqueadas pela avaliação cruzada e serve de referência para todas as demais.
A tabela 4 mostra os índices de regressão Tobit utilizando como variável dependente os índices de eficiência dos vários modelos DEAs apresentados na tabela 2 e como variável independente os insumos apresentados na tabela 1 . Os índices foram obtidos através da utilização software Evews.5.

Tabela 4. Índice de regressão entre os insumos e os índices de eficiências das Escolas

\begin{tabular}{c|c|c|c|c|c|c}
\hline CCR Insumo & BCC Insumo & CCR Produto & BCC Produto & SBM & RAM & CRUZADA \\
\hline $47,57 \%$ & $71.66 \%$ & $49.53 \%$ & $32.72 \%$ & $41.18 \%$ & $64.27 \%$ & $53.21 \%$ \\
\hline
\end{tabular}

Fonte: Índices obtidos através da utilização software Evews.5.

A partir da tabela 4, foi possível calcular o índice de correlação entre os insumos e os índices de eficiência de cada modelo DEA.

Tabela 5. Índice de correlação múltipla entre os insumos e os índices de eficiências das Escolas.

\begin{tabular}{c|c|c|c|c|c|c}
\hline $\begin{array}{c}\text { CCR } \\
\text { INSUMO }\end{array}$ & $\begin{array}{c}\text { BCC } \\
\text { INSUMO }\end{array}$ & $\begin{array}{c}\text { CCR } \\
\text { PRODUTO }\end{array}$ & $\begin{array}{c}\text { BCC } \\
\text { PRODUTO }\end{array}$ & SBM & RAM & CRUZADA \\
\hline 0.6897 & 0.8465 & 0.7045 & 0.5720 & 0.5417 & 0.8018 & 0.7295 \\
\hline
\end{tabular}

Fonte: Índices obtidos através da utilização software Evews.5.

Observa-se, através da tabela 5 , que os modelos BCC insumo, RAM e Avaliação Cruzada apresentaram, de acordo com os dados, melhores índices de relacionamentos com os insumos, evidenciando uma melhor consistência nestes modelos de pesquisas.

Pode-se observar, através da tabela 4, que o modelo BCC insumo é o mais consistente, pois os insumos conseguem explicar $71.66 \%$ dos índices de eficiências das escolas. Embora sendo o modelo mais significativo para este tipo de pesquisa (com dados coletados) apresenta certa desvantagem em relação a avaliação cruzada pelo fato de utilizar muitos pesos iguais a zero para determinar seu índice de eficiência, desta forma gera muitas DMUs eficientes.

O quadro abaixo mostra a análise de regressão Tobit entre os seis insumos e os índices de eficiência do modelo BCC insumos, utilizando o software EViews.5. 
Dependent Variable: Y2

Method: ML - Censored Normal (TOBIT) (Quadratic hill climbing)

Date: 06/10/13 Time: 22:25

Sample: 20012050

Included observations: 50

Left censoring (value) series: 0

Right censoring (value) series: 1

Convergence achieved after 7 iterations

Covariance matrix computed using second derivatives

\begin{tabular}{|c|c|c|c|c|}
\hline & Coefficient & Std. Error & z-Statistic & Prob. \\
\hline $\mathrm{X} 1$ & -0.013835 & 0.009667 & -1.431100 & 0.1524 \\
\hline $\mathrm{X} 2$ & 0.008097 & 0.018407 & 0.439886 & 0.6600 \\
\hline $\mathrm{x} 3$ & -0.002587 & 0.001904 & -1.358593 & 0.1743 \\
\hline $\mathrm{X} 4$ & -0.001780 & 0.001367 & -1.302076 & 0.1929 \\
\hline $\mathrm{X} 5$ & 0.003312 & 0.002187 & 1.514698 & 0.1298 \\
\hline$x 6$ & -0.004108 & 0.001568 & -2.620629 & 0.0088 \\
\hline C & 1.439859 & 0.171793 & 8.381354 & 0.0000 \\
\hline \multicolumn{5}{|c|}{ Error Distribution } \\
\hline SCALE:C(8) & 0.097284 & 0.017650 & 5.511795 & 0.0000 \\
\hline R-squared & 0.716622 & \multicolumn{2}{|c|}{ Mean dependent var } & 0.958780 \\
\hline Adjusted R-squared & 0.669392 & \multicolumn{2}{|c|}{ S.D. dependent var } & 0.085626 \\
\hline S.E. of regression & 0.049234 & \multicolumn{2}{|c|}{ Akaike info criterion } & 0.260384 \\
\hline Sum squared resid & 0.101806 & \multicolumn{2}{|c|}{ Schwarz criterion } & 0.566308 \\
\hline Log likelihood & 1.490392 & \multirow{2}{*}{\multicolumn{2}{|c|}{ Hannan-Quinn criter. }} & 0.376882 \\
\hline Avg. log likelihood & 0.029808 & & & \\
\hline Left censored obs & 0 & \multicolumn{2}{|c|}{ Right censored obs } & 31 \\
\hline Uncensored obs & 19 & \multicolumn{2}{|c|}{ Total obs } & 50 \\
\hline
\end{tabular}

Observa-se, através do quadro acima, que pela regressão Tobit, houve 31 valores censurados a direita, ou seja, maiores que 1. A importância da utilização da análise de regressão Tobit é que os parâmetros são estimados por máxima verossimilhança e não por MQO, fornecendo uma estimativa mais consistente e garantia de $7\left(\hat{\varepsilon}_{i}\right)=0$

\section{CONCLUSÃO}

Atualmente, há uma preocupação geral em diagnosticar o desempenho das escolas públicas. O fraco desempenho dos 
alunos nos processos seletivos das universidades e as dificuldades no seu ingresso no mercado de trabalho têm levado pesquisadores a procurar as causas destes eminentes fracassos e oferecer subsídios para possíveis melhorias na qualidade de ensino.

A Secretaria da Educação e o MEC procuram, através da prova do ENEM, avaliar a qualidade do Ensino Médio sem levar em consideração a situação sócio econômica da clientela e outras variáveis que fazem parte do contexto da escola. É evidente que a influência de muitas variáveis tem um papel relevante no desempenho do aluno.

Observa-se através da aplicação dos vários modelos DEAs, uma realidade preocupante, pois, mesmo os indicadores educacionais mostrando o ensino público abaixo do esperado, a maioria das escolas inclusive as públicas, funcionam dentro de um índice de eficiência. Isto pode ser explicado pelo fato de que a grande maioria das instituições de ensino público apresenta situações e problemas semelhantes.

O grande número de variáveis comuns nas escolas é desfavorável a uma aprendizagem consistente, porém, mesmo assim, as escolas com todas suas diversidades conseguem mostrar certo grau de eficiência.

Embora, neste trabalho, o modelo BCC insumo apresentou-se mais consistente, a avaliação cruzada mostra-se mais eficaz, pois evita que modelo DEA atribua vários pesos zeros para conseguir sua eficiência, o que não é interessante em uma pesquisa educacional.

Observou-se que uma das escolas se destacou, servindo de modelo (Benchmarking), para as escolas ineficientes. Talvez, seria o caso das secretarias ou núcleos de ensino analisar o que de diferente esta escola possui e/ou está desenvolvendo para elaborarem projetos de ensino mais eficazes.

Este trabalho mostrou que a metodologia DEA pode ser um instrumento importante para avaliação periódica das instituições de ensino, auxiliando nas tomadas de decisões e no desenvolvimento de seus projetos.

\section{REFERÊNCIAS}

BANKER, R. D.; CHARNES, A.; COOPER, W. W. Some models for estimating technical and. le inefficiencies in data envelopment analysis. Management Science, v. 30, p. 1078-1092, 1984.

COELLI, T.; RAO, D. S. P.; BALTESE, G. E. An introduction to efficiency and productivity analysis. Massachusetts: KAP, 1998.

CHARNES, A.; COOPER, W. W.; RHODES, E. Measuring efficiency of decision making units. European Journal of Operational Research, v. 2, p. 429-444, 1978.

COOPER, W. W.; PARK, K. S.; PASTOR, J. T. RAM: a range adjusted measure of inefficiency for use with additive models, and relations to other models and measures in 
DEA. Journal of Productivity Analysis, v. 11, p. 5-42, 1999.

DOYLE, J.; GREEN, R. Efficiency and crossefficiency in DEA derivations, meanings and uses. J. Opl. Res. Soc., [S. I.], v. 45, p. 567578, 1994.

KIRJAVAINEN, T.; LOIKKANEN, H. A Efficiency and Productivity in Finnish Comprehensive Schooling 1998-2004. Research Reports, 1995.

RIOS, L. R. Medindo a eficiência relativa das operações dos terminais de contêineres do Mercosul. 2005. Dissertação (Mestrado)Universidade Federal do Rio Grande do Sul, Porto Alegre, RS.

THANASSOULIS, E. A comparison of regression analysis and data envelopment analysis as alternative methods for performance assessments. J. Opl. Res. Soc., v. 44, n. 11, p. 1129-1144, 1993.

TONE, K.; TSUTSUI, M. Dynamic DEA: a slacksbased measure approach. Omega, v. 38, p. 145-156, 2010. 